\title{
Transport Properties of Strongly Correlated Electrons in Quantum Dots Using a Simple Circuit Model
}

\author{
G. B. Martins ${ }^{a}$, C. A. Büsser ${ }^{b}$, K. A. Al-Hassanieh ${ }^{b}$, E. V. Anda ${ }^{c}$, A. Moreo ${ }^{b}$ and E. Dagotto ${ }^{b}$ \\ ${ }^{a}$ Department of Physics, Oakland University, Rochester, MI 48309, USA \\ ${ }^{b}$ University of Tennessee, Knoxville and Condensed Matter Sciences Division, \\ Oak Ridge National Laboratory, Oak Ridge, Tennessee 37831, USA \\ ${ }^{c}$ Departamento de Física, Pontifícia Universidade Católica do Rio de Janeiro, 22453-900, Brazil
}

Received on 08 December, 2005

\begin{abstract}
Numerical calculations are shown to reproduce the main results of recent experiments involving nonlocal spin control in nanostructures (N. J. Craig et al., Science 304, 565 (2004)). In particular, the splitting of the zerobias-peak discovered experimentally is clearly observed in our studies. To understand these results, a simple "circuit model" is introduced and shown to provide a good qualitative description of the experiments. The main idea is that the splitting originates in a Fano anti-resonance, which is caused by having one quantum dot sideconnected in relation to the current's path. This scenario provides an explanation of Craig et al.'s results that is alternative to the RKKY proposal, which is here also addressed.
\end{abstract}

Keywords: Kondo effect; Nanoscopic systems; Correlated electrons

In this Letter, numerical simulations in good agreement with the experiments reported by Craig et al. [1] are presented. The central conclusion of this work is that Fano antiresonance ideas are essential to explain this experimental results. Although the study of transport properties including current noise[6] of a microscopic quantum model is the most common way to understand nanoscopic transport, we will instead explain the experiments using a a very simple "circuit model", where one of the elements is a T-connected QD that has an intrinsic reduction of conductance with varying biases. Fig. 1a depicts the experimental set up used in the measure-

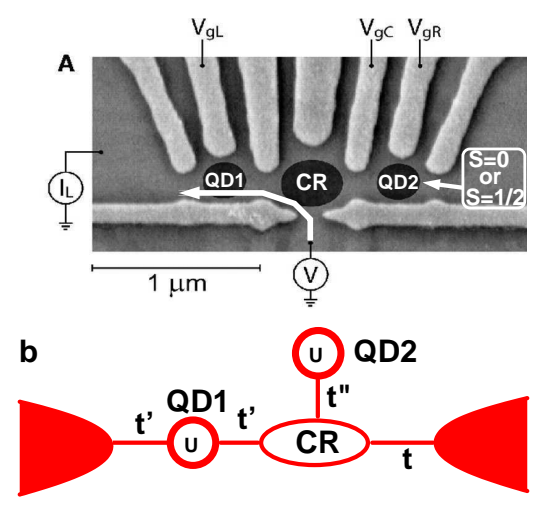

FIG. 1: (a) Experimental setup used in Ref. [1]. (b) Illustration of the model studied in this Letter (see text for details).

ments [1] with the labeling used in this Letter. Figure $1 \mathrm{~b}$ is a schematic representation of the system, introducing two different tunneling parameters (hopping matrix elements $t^{\prime}$ and $t^{\prime \prime}$ ) and the Coulomb repulsion $\mathrm{U}$ in each QD (assumed the same for simplicity). To model this system, the Anderson impurity Hamiltonian is used for both QDs. To calculate the conductance $G$, using the Keldysh formalism [2], a cluster containing the interacting dots and a few sites of the leads is solved exactly[3], the Green functions are calculated, and the leads are incorporated through a Dyson Equation embedding procedure. Details of the embedding have been extensively discussed before[4]. All the results shown were obtained for $U=0.5, t^{\prime}=0.2$, zero-bias, and zero temperature.

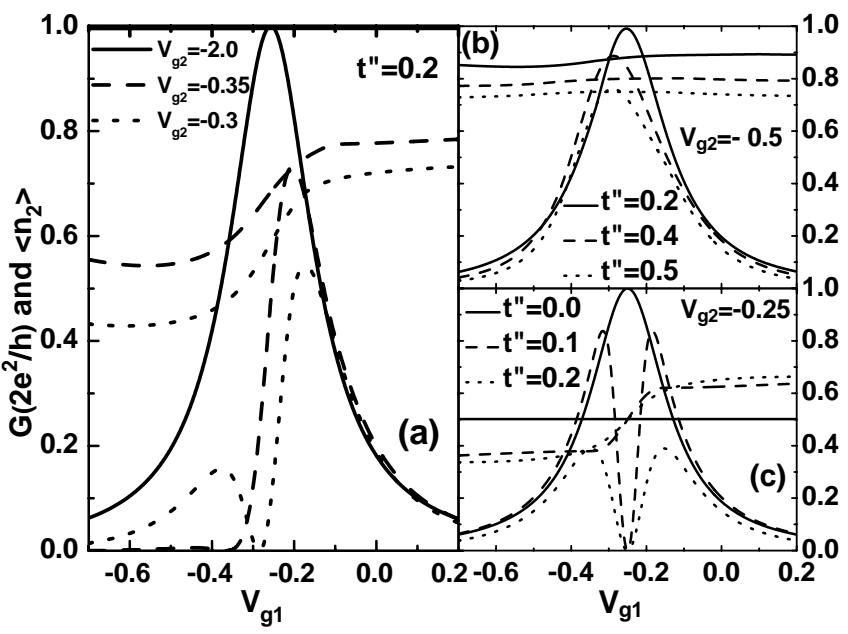

FIG. 2: Numerical results obtained at (a) fixed $t^{\prime \prime}$ and varying $V_{g 2}$, and for fixed $V_{g 2}$ with varying $t^{\prime \prime}$ for two different occupancies of QD2: (b) close to two electrons and (c) close to one electron. See text for details.

In Fig. 2, results for the conductance across QD1 and for the occupancy per spin orientation $\left\langle n_{2}\right\rangle$ of QD2 are presented. In Fig. $2 \mathrm{a}, t^{\prime \prime}=0.2$ and $V_{\mathrm{g} 2}$ varies from -2.0 to -0.3 . For $V_{\mathrm{g} 2}=-2.0$, QD2 is occupied by two electrons $\left(\left\langle n_{2}\right\rangle=1\right)$ and the conductance of QD1 displays the characteristic Kondo behavior reported before [5]. For $V_{\mathrm{g} 2}=-0.35$ the average value of $\left\langle n_{2}\right\rangle$ decreases to $\approx 0.7\left(\approx 1.4\right.$ electrons in QD2) and $\left\langle n_{2}\right\rangle$ 
now depends on $V_{\mathrm{g} 1}$. In addition, $G$ decreases in comparison to the result obtained for $V_{\mathrm{g} 2}=-2.0$. Then, these numerical results are qualitatively in agreement with the experimental results shown in Fig. 2 of Craig et al. [1], namely, by decreasing the occupancy of QD2, from even to odd number of electrons, the zero-bias anomaly (ZBA) in QD1 is suppressed. As $V_{\mathrm{g} 2}$ is further increased $(-0.3)$ a qualitative change occurs: For values of $V_{\mathrm{g} 1}$ where $\left\langle n_{2}\right\rangle \approx 0.5$ (QD2 singly occupied), the conductance of QD1 vanishes and therefore there is a narrow dip in $G$. This splitting of the ZBA is remarkably similar to that observed in Fig. 3A of the experimental results[1]. For finite-temperature calculations, the dip in $G$ will not reach zero, resembling even better the experiments[?].

To further test the similarities between simulations and experiments, in Figs. $2 \mathrm{~b}$ and $2 \mathrm{c}$ results for $G$ and $\left\langle n_{2}\right\rangle$ are shown for fixed $V_{\mathrm{g} 2}$ and different $t^{\prime \prime}$ values. In Fig. 2b, where $V_{\mathrm{g} 2}=-0.5$, as $t^{\prime \prime}$ increases from 0.2 to 0.5 there is only a slight decrease of $G$. This is accompanied by a slight decrease in the average value of $\left\langle n_{2}\right\rangle$, from $\approx 0.9$ to $\approx 0.7$. A more dramatic change is obtained in Fig. 2c, where $V_{\mathrm{g} 2}=-0.25$, and $t^{\prime \prime}$ varies from 0.0 to 0.2 . By increasing $t^{\prime \prime}$ from 0.0 (red curves) to 0.1 , the ZBA is now split in two and $\left\langle n_{2}\right\rangle$ acquires a dependence on $V_{\mathrm{g} 1}$. As $t^{\prime \prime}$ further increases (0.2), the dip becomes wider, the two side-peaks decrease and $G$ still vanishes for $\left\langle n_{2}\right\rangle=0.5$ (one electron in QD2). Our calculations show that, if $\left\langle n_{2}\right\rangle$ varies around 0.5 , the $\operatorname{dip}$ in $G$ is present for all finite values of $t^{\prime \prime}$, with a width proportional to $t^{\prime \prime}$. Comparing the results in Figs. 3A and 3B of Craig et al. [1] with Figs. 2c and $2 \mathrm{~b}$ in this Letter, respectively, one notices a striking similarity: The splitting of the ZBA observed in the experimental results (their Fig. 3A), when the number of electrons in the control QD is odd and the coupling to the central region is increased, is very similar to the $\operatorname{dip}$ in $G$ for all finite- $t^{\prime \prime}$ curves in Fig. 2c (as mentioned above, at finite temperatures, one expects that the dip in $G$ will not reach zero). When the occupancy of QD2 is even (Fig. 3B in the experimental results [1] and Fig. 2b in this Letter), the $G$ dependence on $t^{\prime \prime}$ is much less significant and the splitting of the ZBA does not occur.

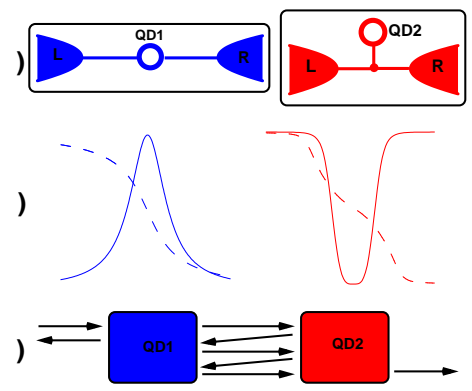

FIG. 3: Schematic representation of the main ideas behind the "circuit model". See text for details.

What is the origin of these results? Below, it will be argued that a qualitative description of the results can be achieved by analyzing the two quantum dots through a so-called 'circuit model'. This model starts with the conductance of each QD calculated separately, as independent elements of a cir- cuit, and then the conductance of the 'complete circuit' is obtained by combining the conductances of the two elements connected in series. Fig. 3 describes schematically the steps involved in this approach. In Fig. 3a, the complete system formed by QD1 and QD2 (shown in Fig. 1b) is divided into two components. QD1 is modeled as a QD connected directly to left (L) and right (R) leads, while QD2 is modeled as a side-connected QD[7]. Fig. 3b shows the respective conductances and occupancies for each independent element vs. gate voltage, and Fig. 3c represents the scattering processes (represented by transmission and reflection amplitudes) that an electron undergoes while moving through the complete 'circuit'. The superposition of all these processes leads to the total transmittance (proportional to the conductance) for the circuit model. This can be calculated in two ways: coherently or incoherently[8]. Since there is no qualitative difference between them, and in order to keep the simplicity of the model, we present the incoherent results. The equation which provides the final transmittance for the processes depicted in Fig. $3 \mathrm{c}$ is

$$
T=\frac{T_{1} T_{2}}{1-R_{1} R_{2}},
$$

where the transmittances $T_{1}$ and $T_{2}$ are proportional to the conductances for QD1 and QD2, as depicted in Fig. 3b, and $R_{1(2)}=1-T_{1(2)}$ are the reflectances. To calculate $T$, one needs to establish how $T_{2}$ depends on $V_{\mathrm{g} 1}$. The natural way to do that is to use the dependence of $\left\langle n_{2}\right\rangle$ on $V_{\mathrm{g} 1}$, as depicted in Fig. 2, and then use the relation between conductance and occupancy, as shown in the red curves in Fig. 3b. In other words, the functional relation can be expressed as $T_{2}=T_{2}\left(\left\langle n_{2}\right\rangle\left(V_{\mathrm{g} 1}\right)\right)$. It is not surprising that in a strongly correlated system like the one being analyzed here, the variation of the gate potential of QD1 will influence the charge occupancy of QD2, and in turn this will influence the conductance through QD1.

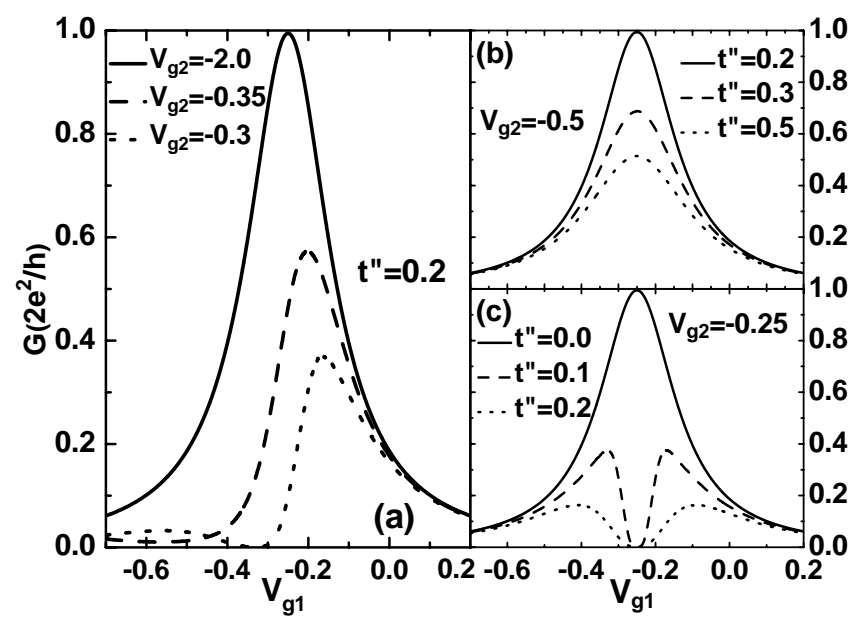

FIG. 4: Same as in Fig. 2, but now using the 'circuit model' for the calculations.

In Fig. 4, conductance results using Eq.(4) are shown for the same parameters as in Fig. 2. Although the quantitative 
agreement varies, there is good overall qualitative agreement. All the trends are correctly reproduced and some of the details are quite similar, such as for example the asymmetric shape of the curves at higher values of $V_{\mathrm{g} 2}(-0.35$ and -0.3$)$ in Fig. 4a. The success of the circuit model implies that the dip in $G$ arises from the Fano anti-resonance which cancels the conductance of QD2 (red solid curve in Fig. 3b). The Fano antiresonance can be seen as a destructive interference process between two different trajectories an electron can take on its way to QD1: it can cross the CR without passing through QD2; or it can visit QD2, return to the $\mathrm{CR}$ and then proceed to QD1 [7].

In the following, just a brief account is given of the importance of the RKKY interaction in the experimental setup being discussed. The authors found out that (as expected) there is a coupling between QD1 and QD2 which seems to have the characteristics of an RKKY interaction. However, by redoing the conductance calculations with the Hubbard inter- action in QD1 set to zero, where any long range interaction between QD1 and QD2 vanishes, the splitting of the conductance peak is not affected, indicating that its origin can be fully ascribed to the interference process described above. In summary, the numerical results qualitatively reproduce the main aspects of important recent experiments [1] involving nonlocal spin control in nanostructures. The main result is that the splitting observed in the ZBA is caused by a cancellation in the conductance due to a destructive interference. This so-called Fano anti-resonance has its origin in one of the dots being side-connected to the current's path. A simple 'circuit model' qualitatively reproduces the experiments and offers an alternative to a purely RKKY interpretation of the results, underscoring that a laboratory realization of the two-impurity Kondo system should avoid any geometry susceptible to a Fano antiresonance.

C.B., K. A., A. M., and E. D. are supported by NSF grant DMR 0454504. G. M. by an OU internal grant.
[1] N. J. Craig , J. M. Taylor, E. A. Lester, C. M. Marcus, M. P. Hanson, and A. C. Gossard, Science 304, 565 (2004).

[2] Y. Meir, N. S. Wingreen, and P. A. Lee, Phys. Rev. Lett. 66, 3048 (1991)

[3] Results shown include one site on each side. Calculations with more sites were done, but no significative size-effects were observed.

[4] See V. Ferrari, G. Chiappe, E. V. Anda, and M. A. Davidovich, Phys. Rev. Lett. 82, 5088 (1999).
[5] Removing QD2 results in essentially the same conductance as the one for $V_{\mathrm{g} 2}=-2.0$.

[6] A. L. Yeyati, F. Flores, and E. V. Anda, Phys. Rev. B 47, 10543 (1993).

[7] R. Franco, M. S. Figueira, and E. V. Anda, Phys. Rev. B 67, 155301 (2003); M. Sato et al., cond-mat/0410062.

[8] S. Datta, Electronic Transport in Mesoscopic Systems, Londo: Cambridge University Press, 1995. 